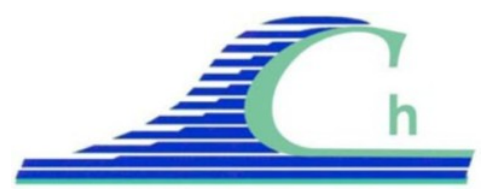

XII ${ }^{\text {ìmes }}$ Journées Nationales Génie Côtier - Génie Civil

Cherbourg, 12-14 juin 2012

DOI:10.5150/jngcgc.2012.105-P @ Editions Paralia CFL



\title{
Prévision opérationnelle des niveaux de la mer, surcotes et décotes sur les côtes de la Manche et de l'Atlantique
}

\section{Lucia PINEAU-GUILLOU ${ }^{1}$, Sébastien THEETTEN ${ }^{1}$, Franck DUMAS ${ }^{1}$, Fabrice LECORNU ${ }^{1}$, Déborah IDIER ${ }^{2}$}

\section{IFREMER, Centre de Brest,}

Technopôle de Brest Iroise, BP 70, 29280 Plouzané, France.

Lucia.Pineau.Guillou@ifremer.fr ; Sebastien.Theetten@ifremer.fr

Franck.Dumas@ifremer.fr ; Fabrice.Lecornu@ifremer.fr

\section{BRGM}

3 avenue C. Guillemin, 45060 Orléans Cedex 2, France.

Deborah.Idier@brgm.fr

\section{Résumé :}

Le système pré-opérationnel PREVIMER fournit des observations et des prévisions côtières sur les trois façades métropolitaines Manche, Atlantique et Méditerranée. Il fournit en particulier les prévisions de courants, niveaux de la mer, surcotes et décotes et vagues. L'objectif de l'étude est de mettre en place un système de modélisation des ondes longues (2DH) haute résolution (une centaine de mètres) sur l'ensemble des côtes métropolitaines. Les expériences menées dans le cadre de PREVIMER ont mis en évidence l'importance d'une meilleure prise en compte de l'action du vent et des vagues - par l'amélioration de la paramétrisation du coefficient de traînée en surface - et du wave set-up. Ainsi, ces modèles à haute-résolution intègrent ces dernières avancées, avec en particulier la prise en compte de la rugosité de la mer en fonction de l'âge des vagues. Comme étape préliminaire, les méthodes de calculs sont ici présentées, ainsi que des premières validations de marée et surcotes modélisées, en particulier à La Rochelle-La Pallice, et pour l'évènement Xynthia (février 2010). Enfin, une analyse de données est effectuée pour mieux quantifier les variations locales de surcotes pendant l'évènement Xynthia, entre le large et le marégraphe de La Rochelle-La Pallice.

\section{Mots-clés :}

Hydrodynamique côtière - Modélisation numérique - Système opérationnel - Niveau de la mer - Surcotes - Coefficient de trainée - Wave set-up

\section{Introduction}

Les événements tels que la tempête Xynthia qui a frappé les côtes métropolitaines le 28 février 2010, nous rappellent que la prévision des surcotes et des niveaux d'eau est un outil indispensable pour la prévention des risques de submersion marine. Mais le développement de modèles de niveaux et courants répond également à de nombreux autres besoins, comme : 
- l'amélioration des modèles de vagues de PREVIMER en prenant en compte l'influence des courants sur les vagues,

- l'aide à la prédiction de la présence ou l'absence de certains habitats (herbiers, laminaires, .....). En effet, la corrélation entre les données d'observation de présence ou absence de certains habitats et les paramètres physiques en relation avec leur développement (e.g. les courants) permet de mettre en place des modèles statistiques (e.g. modèles de niches écologiques) ; ces modèles statistiques permettent ensuite de prédire les habitats en fonctions des conditions environnementales climatologiques ;

- la fourniture de conditions aux limites pour le développement de modèles littoraux, de résolution plus fine (de l'ordre de quelques dizaines de mètres).

Aussi, le système pré-opérationnel PREVIMER fournit des observations et des prévisions côtières sur les trois façades métropolitaines Manche, Atlantique et Méditerranée. Il fournit en particulier les prévisions de niveau d'eau, surcotes et décotes et vagues. L'objectif de l'étude est de mettre en place des modèles opérationnels 2DH à haute résolution spatiale (de l'ordre de $100 \mathrm{~m}$ ) et temporelle $(15 \mathrm{mn}$ ) sur les côtes de la Manche et de l'Atlantique, permettant de calculer les courants barotropes, les niveaux d'eau, les surcotes et les décotes.

L'article présente la mise en place de tels modèles, l'amélioration de leur paramétrisation et une première quantification de la variabilité spatiale des surcotes autour de La Rochelle.

\section{Mise en place des modèles 2D}

\subsection{Emprise}

Afin de reproduire la dynamique des surcotes, il est nécessaire de disposer d'un modèle suffisamment étendu vers le Nord et l'Ouest, pour prendre en compte les passages dépressionnaires qui s’y développent, générant des surcotes se propageant en Manche et dans le Golfe de Gascogne. Les modèles mis en place sont donc des modèles emboîtés (emprises ; voir figure 1) de résolution :

- $2 \mathrm{~km}$ pour la grande emprise, de rang 0, s'étendant jusqu'en Islande,

- 700 m pour le modèle intermédiaire, de rang 1, couvrant la Manche et le Golfe de Gascogne,

- 250 m actuellement pour les cinq modèles de rang 2, mais avec une évolution prévue vers une résolution plus fine. 


\section{XII ${ }^{\text {èmes }}$ Journées Nationales Génie Côtier - Génie Civil \\ Cherbourg, 12-14 juin 2012}

(a)

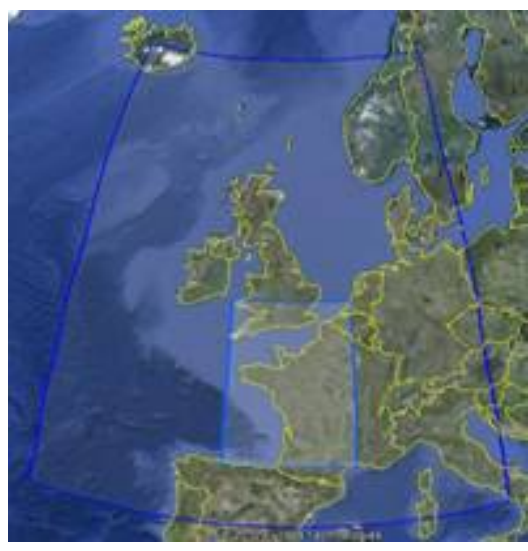

(b)

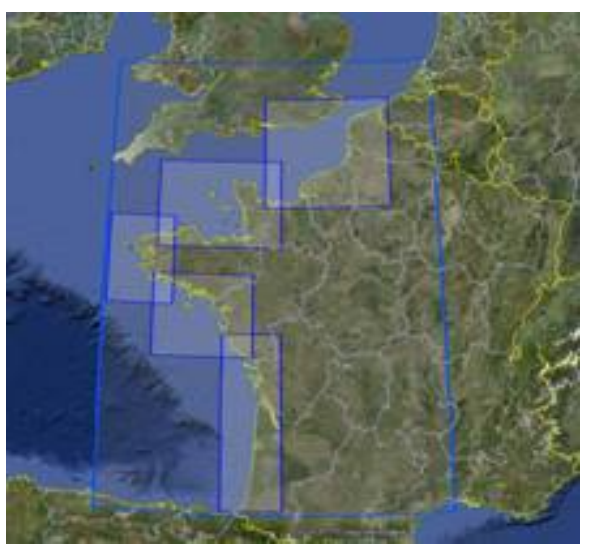

Figure 1. Extension des modèles : (a) rangs 0 et 1 (b) rangs 1 et 2.

\subsection{Méthodologie de calcul des surcotes}

La méthodologie de calcul des surcotes a été développée dans le cadre du projet PREVIMER Phase I (ROMIEU \& IDIER, 2007) et est présentée figure 2. Les surcotes sont calculées en retranchant aux hauteurs issues d'une simulation avec météo les hauteurs issues d'une simulation sans météo. Les rangs 0 et 1 servent uniquement à calculer les surcotes instantanées à la frontière des rangs 2 ; ces rangs 2 prennent pour conditions limites les surcotes issues du rang 1 et les niveaux de marée issus du modèle de marée cstFRANCE du SHOM (LE ROY \& SIMON, 2003).

Cette méthode permet d'obtenir une marée précise au niveau des rangs 2, en particulier dans les zones où la marée est complexe et les ondes d'interaction importantes comme par exemple le Golfe Normand-Breton où bien les Pertuis Charentais.

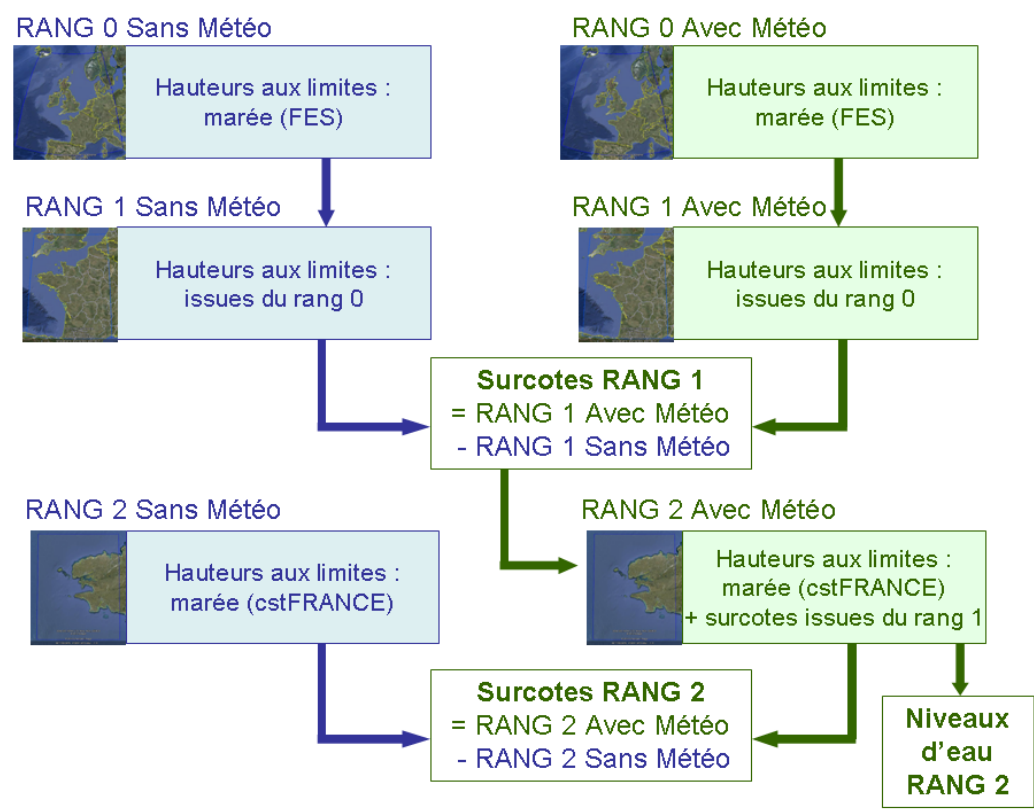

Figure 2. Méthodologie de calcul des niveaux d'eau et surcotes modélisées. 


\subsection{Données d'entrée}

La bathymétrie du modèle est issue des modèles numériques de terrain (MNT) disponibles à l'Ifremer, en particulier le MNT à 100 m SHOM-Ifremer. Les conditions aux limites ouvertes sont prescrites à l'aide de la solution FES2004 (LYARD et al., 2006) au rang 0 et la solution cstFRANCE du SHOM (LE ROY \& SIMON, 2003) pour les rangs 2. Les modèles opérationnels dans PREVIMER sont actuellement forcés par les prévisions à 6h d'ARPEGE. Les modèles en cours de développement seront forcés par les prévisions atmosphériques à haute résolution de Météo-France (ARPEGE haute résolution et AROME à $1 \mathrm{~h}$ ) actualisées quatre fois par jour. Ces nouveaux modèles seront également forcés par les prévisions du Centre Européen de Prévision à moyen terme pour disposer d'une seconde solution.

\subsection{Procédure de validation}

La validation des modèles a été réalisée sur des éléments recueillis en février 2010, pendant la tempête Xynthia (PINEAU-GUILLOU et al, 2010). Elle consiste à :

- comparer la marée modélisée et la marée prédite,

- comparer le niveau total (marée + surcote) modélisé et mesuré,

- comparer les surcotes modélisées et mesurées,

- comparer les courants modélisés et mesurés en morte-eau et vive-eau moyenne.

La marée modélisée est comparée aux prédictions de marée. Ces prédictions sont basées sur l'analyse des hauteurs mesurées toutes les 10 minutes (REFMAR) uniquement sur la période correspondant aux marégraphes numériques, pour s’assurer de la qualité des données (par rapport aux données plus anciennes). Cette période est généralement de plusieurs dizaines d'années, ce qui est suffisant pour une bonne précision de l'analyse harmonique. Les composantes harmoniques et les prédictions sont calculées à partir du logiciel mas développé par le SHOM. Un exemple de comparaison entre la marée modélisée par les différents rangs et la marée prédite est présentée figure 3 : le rang 2 donne les meilleurs résultats.

Les surcotes mesurées sont calculées en retranchant à l'observation les prédictions précédemment effectuées. Un exemple de comparaison entre les surcotes modélisées (avec un coefficient de Charnock variable cf. 3.2) par le rang 0 forcé par ARPEGE 3h fusionné avec AROME, et celles mesurées est présenté figure 4.

\section{Amélioration de la paramétrisation des modèles}

\subsection{Forçages météorologiques}

L'amélioration des forçages météorologiques, permet d'améliorer significativement les résultats. Les premiers résultats montrent qu'à La Rochelle, par rapport au forçage avec ARPEGE 6h, le forçage avec ARPEGE 3h améliore le pic de surcote de $12 \mathrm{~cm}$, et celui avec ARPEGE 3h fusionné avec AROME améliore le pic de surcote de $20 \mathrm{~cm}$. 


\section{XII ${ }^{\text {èmes }}$ Journées Nationales Génie Côtier - Génie Civil \\ Cherbourg, 12-14 juin 2012}

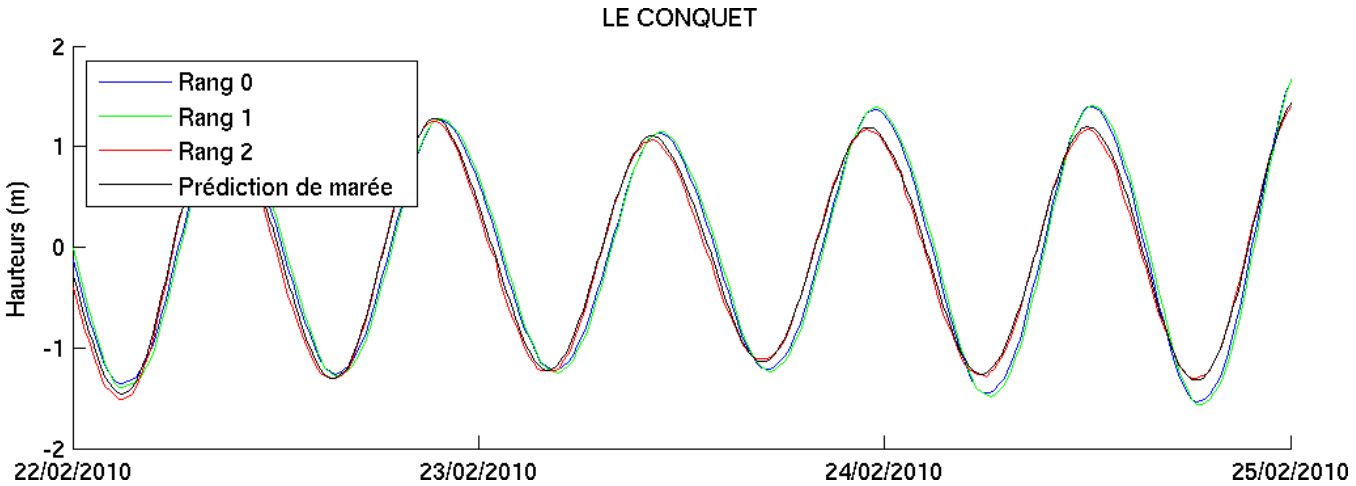

Figure 3. Exemple de comparaison de la marée issue des rangs 0, 1 et 2 et de la prédiction au Conquet.
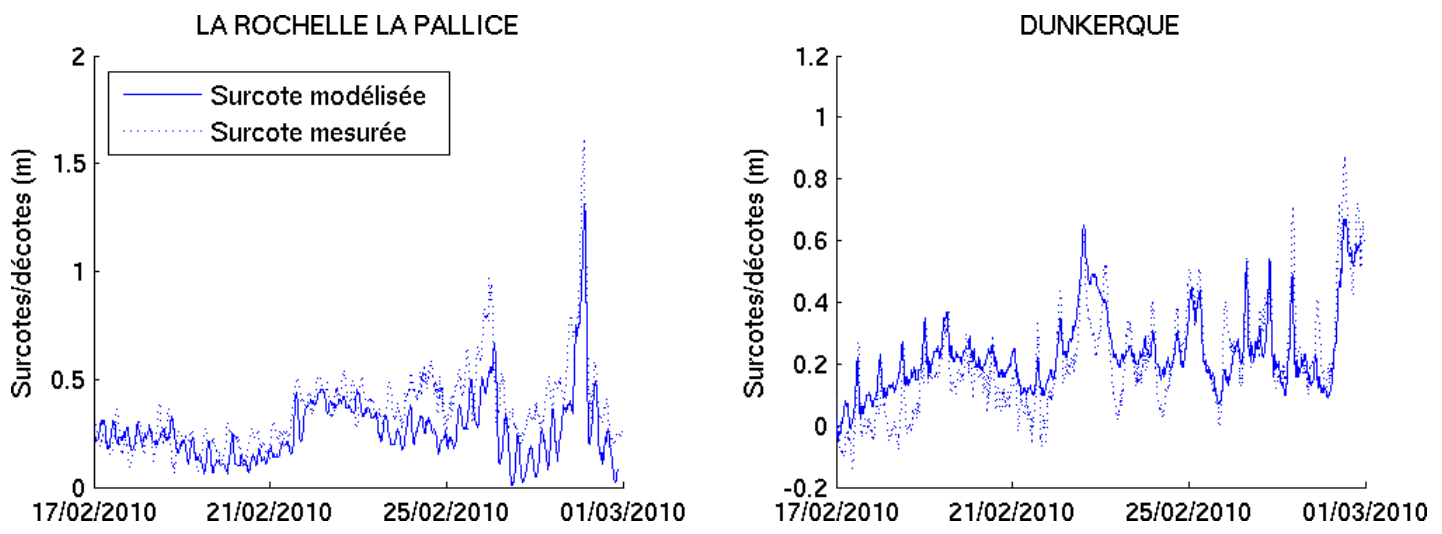

Figure 4. Exemple de comparaison des surcotes modélisées (rang 0, ARPEGE $3 h$ fusionné avec AROME) et mesurées.

\subsection{Action du vent et coefficient de traînée}

Le coefficient de traînée actuellement utilisé pour les modèles opérationnels est constant et égal à 0.0016. La formulation de Charnock (CHARNOCK, 1955) avec un coefficient constant (0.014) permet d'améliorer l'estimation de la surcote pendant Xynthia (figure 5). La prise en compte de l'action des vagues permet encore d'améliorer cette paramétrisation : en utilisant le coefficient de Charnock variable issu des modèles de vagues WaveWatchIII ${ }^{\circledR}$ implémentés dans PREVIMER, l'amélioration de l'estimation des surcotes atteint une dizaine de cm pendant la tempête Xynthia à La Rochelle. L'amélioration totale pendant Xynthia entre un coefficient de traînée constant et une formulation de Charnock à coefficient variable atteint une vingtaine de cm (18 cm à La Rochelle). Ce résultat est consistant avec ceux obtenus dans le cadre du projet PREVIMER-surcotes (IDIER et al., 2010a, 2010b, 2012) et ceux obtenus par (BERTIN et al, 2012). 


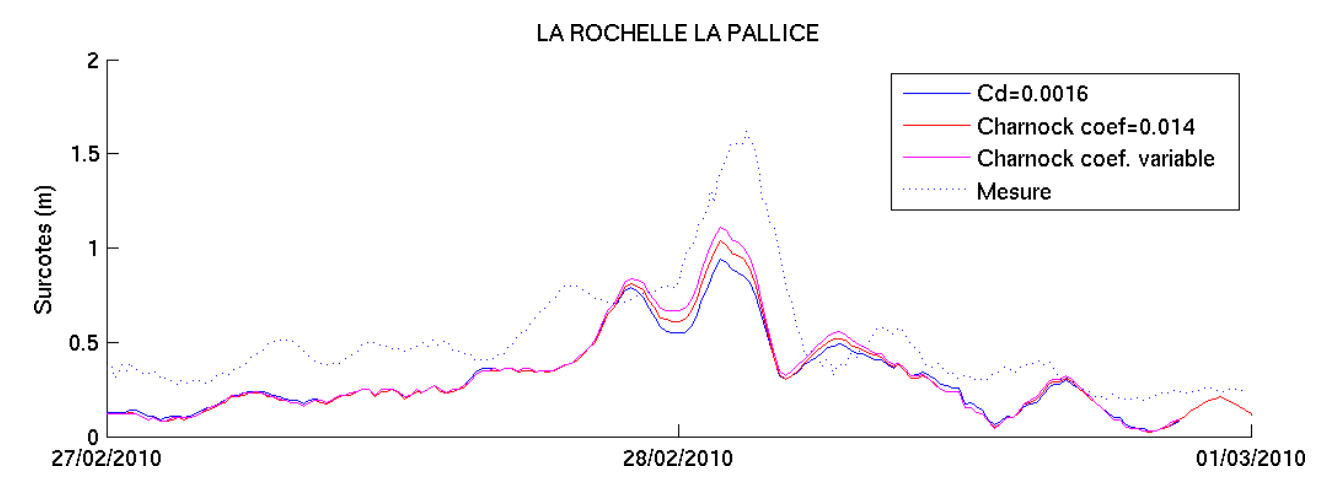

Figure 5. Influence de la paramétrisation du coefficient de traînée sur les surcotes modélisées (rang 0, ARPEGE 6h).

\subsection{Variabilité spatiale des surcotes et wave set-up}

Du 3 février au 24 mars 2010, deux marégraphes étaient mouillés par le SHOM pour ses propres besoins hydrographiques : le marégraphe M1 au large de l'île de Ré et celui de La Cotinière sur l'île d’Oléron (figure 6.a). L'exploitation des données du marégraphe M1 permet d'estimer le wave set-up pendant Xynthia au niveau du port de la Rochelle. Les surcotes de pleine et basse mer ont été calculées en retranchant aux observations de pleine et basse mer des prédictions de pleine et basse mer, issues de l'analyse des données. La durée d'enregistrement du marégraphe M1 trop courte pour permettre une analyse harmonique complète (inférieure à 2 mois) a été analysée à l'aide d'une concordance par espèces effectuée avec les données de La Rochelle (SIMON, 2007). La concordance entre les surcotes de pleine et basse mer au niveau de La Rochelle et du marégraphe M1 est présentée figure 6.b. La droite rouge représente une régression sur l'ensemble des surcotes, hors Xynthia. Les surcotes à La Rochelle sont globalement proportionnelles à celle du marégraphe M1, sauf pendant la tempête Xynthia. La surcote à la Rochelle est alors supérieure de plusieurs dizaines de cm (30 à $40 \mathrm{~cm}$ ) à la surcote de M1 multipliée par le coefficient de proportionnalité de la droite de régression, précédemment obtenue (0.92). Cette différence peut s’expliquer par différents phénomènes, dont les principaux sont :

- la présence éventuelle de set-up au niveau de La Rochelle pendant Xynthia, due au déferlement des vagues,

- l'action locale du vent pendant Xynthia.

L'action du vent est plus importante que celle de la pression atmosphérique par petits fonds (FLATHER, 2001). Ainsi, l'effet de la pression atmosphérique va être a priori identique entre La Rochelle et M1, alors que l'effet du vent peut engendrer une surcote plus importante à La Rochelle qu'en M1.

Des études sont actuellement en cours pour déterminer la part du wave set-up et celle de l'action locale du vent dans cette surcote, en particulier à partir de l'exploitation du modèle de rang 2 couvrant les Pertuis Charentais. En outre, l'estimation du set-up via des formulations empiriques comme celles de Stockdon (STOCKDON et al., 2006) 


\section{XII ${ }^{\text {èmes }}$ Journées Nationales Génie Côtier - Génie Civil \\ Cherbourg, 12-14 juin 2012}

dans les systèmes opérationnels à partir des hauteurs significatives issues des modèles WaveWatchIII $^{\circledR}$ de PREVIMER, et de la connaissance des pentes de plage est en cours d'étude.

(a)

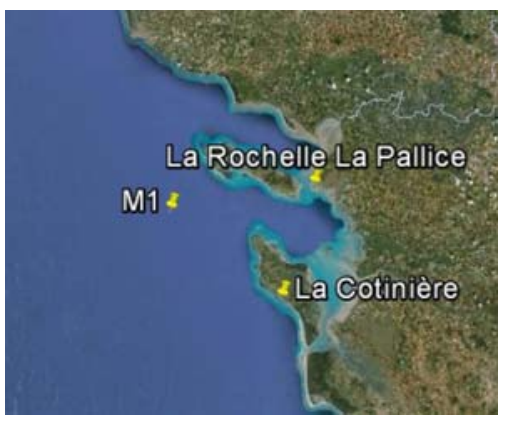

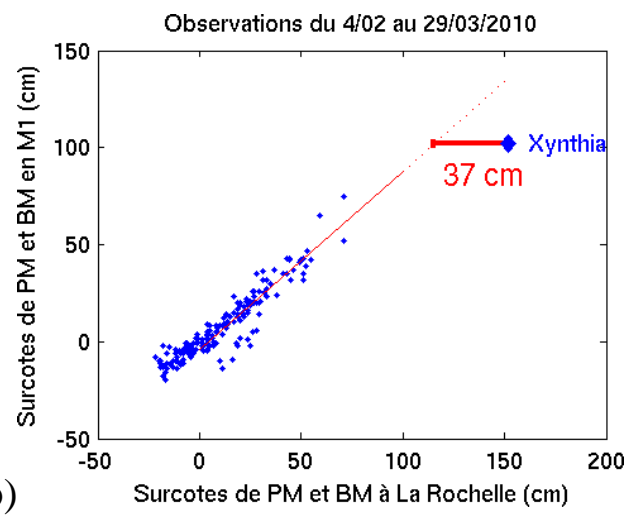

Figure 6. (a) Localisation du marégraphe M1 et (b) Concordance entre les surcotes à la Rochelle et les surcotes du marégraphe M1 du 4 février au 29 mars 2010.

\section{Conclusions}

Les modèles en cours de développement constituent une amélioration par rapport à la chaîne opérationnelle existante : meilleure résolution spatiale, meilleure prise en compte du vent, meilleurs forçages météorologiques. De nombreux développements sont encore en cours, en particulier concernant la prise en compte du wave set-up.

Ces modèles seront intégrés dans le système pré-opérationnel PREVIMER courant 2012 et utiliseront plusieurs forçages météorologiques : les prévisions atmosphériques à haute résolution de Météo-France (ARPEGE haute résolution et AROME actualisées quatre fois par jour), ainsi que les prévisions du Centre Européen de Prévision Météorologique. Ces nouveaux modèles fourniront des prévisions haute résolution du niveau de la mer, des surcotes et décotes, et des courants.

\section{Remerciements}

Les hauteurs mesurées au niveau des marégraphes côtiers ont été obtenues grâce au site REFMAR (http://refmar.shom.fr/) du SHOM. Les hauteurs d'eau au niveau du marégraphe M1 ont été fournies par le SHOM dans le cadre de PREVIMER. Les forçages météorologiques ont été fournis par Météo-France dans le cadre de PREVIMER. Les auteurs remercient également les participants au comité de pilotage du projet PREVIMER-surcotes (Ifremer, BRGM, Météo-France, SHOM).

\section{Références bibliographiques}

BERTIN X., BRUNEAU N., BREILH J.-F., FORTUNATO A.B., KARPYTCHEV M. (2012). Importance of wave age and resonance in storm surges: The case Xynthia, Bay of Biscay. Ocean Modelling, vol. 42, pp 16-30. doi:10.1016/j.ocemod.2011.11.001 
Thème 7 - Océanographie opérationnelle et situations extrêmes

CHARNOCK H. (1955). Wind stress on a water surface. Quart. J. Roy. Meteor. Soc., vol. 81, pp 639-640. doi:10.1002/qj.49708135027

FLATHER R.A. (2001) Storm surges, Encyclopedia of Ocean Sciences (ed. : Steele, J. H., S. A. Thorpe, and K. K. Turekian), Academic, San Diego, Calif, pp 2882-2892.

IDIER D., KRIEN Y., PEDREROS R., DESRAMAUT N., PINEAU-GUILLOU L., DUMAS F. (2010a). Système de prévision de surcotes en Manche/Atlantique et Méditerranée : Etude bibliographique. Rapport BRGM/RP-58918-FR, 92 p.

IDIER D., KRIEN Y., THIEBOT J., PEDREROS R., avec la collaboration de DUMAS F., LECORNU F., PINEAU-GUILLOU L., OHL P., PARADIS D. (2010b). Système de prévision de surcotes en Manche/Atlantique et Méditerranée : Sites sélectionnées et collecte-traitement des données. Rapport BRGM/RP-59039-FR, 133 p.

IDIER D., DUMAS F., MULLER H., PEDREROS R., THIEBOT J., YATES M., LECORNU F., PINEAU-GUILLOU L., CREACH D. et PARADIS D. (2012) Modélisation des surcotes sur les côtes françaises, manche et atlantique, Congrès SHF : "Evènements extrêmes fluviaux et maritimes", Paris, 1-2 février 2012.

LE ROY R., SIMON B. (2003). Réalisation et validation d'un modèle de marée en Manche et dans le Golfe de Gascogne. Application à la réalisation d'un nouveau programme de réduction des sondages bathymétriques. Rapport SHOM n002/03.

LYARD F., LEFEVRE F., LETELLIER T., FRANCIS O. (2006). Modelling the global ocean tides: modern insights from FES2004. Ocean Dynamics, vol. 56, pp 394-415 . doi:10.1007/s10236-006-0086-x

PINEAU-GUILLOU L., LATHUILIERE C., MAGNE R., LOUAZEL S., CORMAN D., PERHERIN C. (2010). Caractérisation des niveaux marins et modélisation des surcotes pendant la tempête Xynthia. XI ${ }^{\text {èmes }}$ Journées Nationales Génie Civil - Génie Côtier, Sables d’Olonne, pp 625-634. doi:10.5150/jngcgc.2010.073-P

ROMIEU E., IDIER D. (2007). Previmer - Opérationnalisation de modèles hydrodynamiques régionaux - Délivrables M1 et M2 : Sorties et lecture des résultats à tous les n pas de temps de calcul \& Optimisation ondes de tempêtes - onde de marée. Rapport BRGM/RP-55862-FR, 83 p.

SIMON B. (2007). La marée océanique côtière. Institut Océanographique, Paris, 544 p. STOCKDON H.F., HOLMAN R.A., HOWD P.A., SALLENGER JR A.H. (2006). Empirical parameterization of setup, swash, and runup. Coastal Eng., vol. 53, pp. 573588. doi:10.1016/j.coastaleng.2005.12.005 